\title{
BMJ Open Study protocol for a randomised controlled trial of cognitive processing therapy for post-traumatic stress disorder among Japanese patients: the Safety, Power, Intimacy, Esteem, Trust (SPINET) study
}

Masaya Ito, ${ }^{1}$ Masaru Horikoshi, ${ }^{2}$ Patricia A Resick, ${ }^{3}$ Akiko Katayanagi, ${ }^{2}$ Mitsuhiro Miyamae, ${ }^{2,4}$ Yuriko Takagishi, ${ }^{5}$ Yoshitake Takebayashi, ${ }^{2,6}$ Ayako Kanie, ${ }^{2}$ Naotsugu Hirabayashi, ${ }^{7}$ Toshiaki A Furukawa ${ }^{8}$

To cite: Ito M, Horikoshi M, Resick PA, et al. Study protocol for a randomised controlled trial of cognitive processing therapy for post-traumatic stress disorder among Japanese patients: the Safety, Power, Intimacy, Esteem, Trust (SPINET) study. BMJ Open 2017;7:e014292. doi:10.1136/ bmjopen-2016-014292

- Prepublication history and additional material for this paper are available online. To view these files please visit the journal online (http://dx.doi. org/10.1136/bmjopen-2016014292).

Received 15 September 2016 Revised 19 May 2017 Accepted 7 June 2017

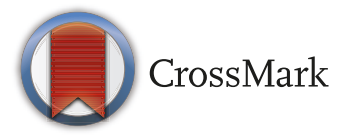

For numbered affiliations see end of article.

Correspondence to Dr Masaya Ito; masayait@ncnp. go.jp, masayaito@me.com

\section{ABSTRACT \\ Introduction Cognitive processing therapy (CPT) is widely regarded as a safe and effective first-line treatment for individuals with post-traumatic stress disorder (PTSD); however, no comparative studies have been conducted to examine the treatment outcomes in an Asian population. The aim of the present trial is to investigate the efficacy of CPT (individual format) as a treatment for PTSD in a population of Japanese patients.}

Methods and analysis A 16-week, single-centre, assessor-masked, randomised, parallel-group superiority trial has been designed to compare the efficacy of CPT in conjunction with treatment as usual (mostly pharmacotherapy and clinical monitoring) versus treatment as usual alone. The Clinician-Administered PTSD Scale for the Diagnostic and Statistical Manual of Mental Disorders, Fifth Edition (DSM-5) will be our primary outcome measure of the post-traumatic stress symptoms at 17 weeks, whereas the PTSD Checklist for DSM-5 and determination of the operationally defined responder status will be used to assess the secondary outcomes. An estimated sample size of 29 participants in each group will be required to detect an expected effect size of 1.4 (95\% Cl 0.85 to 1.95). Ethics and dissemination The institutional review board at the National Center of Neurology and Psychiatry in Japan approved this study. The results of this clinical trial will be presented at conferences and disseminated through publication in a peer-reviewed journal.

Trial registration number UMIN000021670 (registered on 1 April 2016).

\section{INTRODUCTION}

\section{Background and rationale}

Experiencing a traumatic event can lead to a reduced quality of life and may negatively affect an individual's physical and mental health. In Japan and many other regions around the world, individuals are exposed to a vast array of potentially traumatic
Strengths and limitations of this study

- This is the first randomised controlled trial of the newest version of individual cognitive processing therapy, which does not require the patient to provide a written account of the trauma, in a nonWestern context.

- This is the largest randomised controlled trial to be performed in a Japanese clinical setting for patients with post-traumatic stress disorder.

- Major limitations include no active treatment control group and no restriction of pharmacotherapy changes during the intervention period.

situations, including natural disasters, physical and sexual assault, intimate partner violence, child abuse, among others. For example, approximately 33000 Japanese individuals per year are severely injured or killed due to criminal activities, whereas another 9000 fall victim to rape or sexual assault. ${ }^{1}$ Additionally, more than 15000 people died because of the Great East Japan Earthquake in 2011. Experiencing such events may lead to the development of post-traumatic stress disorder (PTSD), a debilitating mental disorder characterised by intrusion symptoms that are associated with the traumatic event, as well as avoidance of triggering situations, alterations in cognition and mood and hyperarousal or reactivity. ${ }^{2}$ Research has revealed that PTSD leads to psychiatric comorbidities, an increased risk of suicide and a variety of negative health outcomes. ${ }^{3}{ }^{4}$ Although the prevalence of PTSD in the Japanese population is relatively low (an estimated annual prevalence of $0.4 \%^{5}$ ) in comparison with that 
of the US population (annual prevalence of $4.7 \%$ ), care and treatment of individuals with PTSD has been far from sufficient in Japan.

Systematic reviews, meta-analyses and treatment guidelines have consistently reported that trauma-focused cognitive behavioural therapies (CBTs) are the treatment of choice for individuals with severe PTSD. ${ }^{6-9}$ Among the forms of CBT that have been employed, prolonged exposure therapy (PE) and cognitive processing therapy (CPT), both of which have been recommended and adapted for the treatment of US military veterans, are two of the strongest evidence-based treatments for PTSD in terms of safety and efficacy. ${ }^{10}$ Although both treatments are classified as CBT, PE focuses on exposure to fearful memories associated with the traumatic event, whereas CPT focuses on cognitive reappraisal of the meaning of the traumatic events and their consequences.

The latest systematic review reported the effect sizes of $\mathrm{PE}$ and CPT in comparison with wait list or treatment as usual (TAU) as having standardised mean differences of $-1.27(95 \%$ CI -1.54 to $-1.00 ; \mathrm{k}=7, \mathrm{n}=387)$ and $-1.40(95 \%$ CI -1.95 to $-0.85 ; \mathrm{k}=4, \mathrm{n}=299$ ), respectively. ${ }^{8}{ }^{9}$ Research has also revealed that CPT and PE are effective in treating depression, suicidal ideation and sleep disturbances, in addition to reducing the annual costs that are associated with providing mental health services. ${ }^{11}$ Further, CPT is highly effective for managing dissociative symptoms, improving social adjustment and reducing the chances of experiencing intimate partner violence in the future. ${ }^{12}$

In traditional CPT, now called CPT+A (ie, CPT with written accounts), ${ }^{13}$ the therapist asks the client to write and subsequently read aloud a detailed account of the traumatic experience. The ultimate goals of CPT $+\mathrm{A}$ are to break patterns of avoidance and to enable the client to modify his or her own cognitive distortions and effectively process the associated emotions. The newest version of CPT eliminates the use of written accounts, placing more emphasis on cognitive processing through patient-therapist interaction and patient engagement in the cognitive work. ${ }^{13}$ The final phase of both treatment programmes focuses on five conceptual areas: safety, power/control, intimacy, esteem and trust (SPINET). While some research has suggested that both $\mathrm{CPT}+\mathrm{A}$ and CPT are effective treatments for the symptoms of PTSD, only four randomised controlled trials (RCTs) have demonstrated the efficacy of the newer version of CPT in comparison with TAU or active psychotherapeutic treatment (one using the individual format and three using the group format). ${ }^{14-17}$ Although some studies have examined the efficacy of CPT $+\mathrm{A}$ or CPT outside of Western cultural settings, no comparative clinical trial using the individual format of CPT has been conducted in an Asian population..$^{15} 1819$

Because both patients and therapists are reluctant to directly remember or confront the details of a traumatic experience using techniques similar to imaginal exposure as PTSD treatments, determining the efficacy of cognitive-only CPT is crucial. ${ }^{20}{ }^{21} \mathrm{~A}$ recent study by Larsen $e t$ $a t^{22}$ revealed that CPT exhibited lower levels of symptom exacerbation $(14.7 \%)$ during treatment than did both CPT $+\mathrm{A}(28.6 \%)$ and PE (20.0\%). Moreover, the drop-out rate was reportedly lower for CPT (22\%) than it was for $\mathrm{CPT}+\mathrm{A}(34 \%){ }^{16}$

The present SPINET study aims to investigate the safety and efficacy of the latest versions of CPT for the treatment of PTSD in a Japanese population in comparison with more commonly used treatment options. There are three important aspects to note when discussing the design of this RCT. The first is the lack of treatment and treatment evidence for PTSD among the Japanese population. Only one small Japanese RCT reported the efficacy of PE in comparison with that of TAU $(n=24$, standardised mean difference post-treatment $=0.95$ ), and this prior study excluded patients who had experienced domestic violence or childhood trauma. ${ }^{23}$ Because PE is covered by the Japanese Ministry of Health, Labour and Welfare, as are more traditional treatments involving selective serotonin reuptake inhibitors such as paroxetine and sertraline, CPT may provide an alternative treatment option for individuals who have not responded to PE or pharmacological interventions. Second, as the national CBT training standards in Japan are based on Beck's cognitive theory of depression, clinical implementation of CPT may be more appropriate because CPT itself was developed according to this same theory. ${ }^{24}$ Studies involving US military veterans also indicated that the degree of CPT implementation is correlated with the clinical experience and existing beliefs (eg, 'cognitive therapy is clinically effective') of the therapist $(\mathrm{r}=0.28, \mathrm{p}<0.09) .{ }^{25}$ Hence, CPT administered by trained Japanese clinicians is expected to be highly effective for improving the clinical outcomes of patients with PTSD. Third, it has been suggested that patients with PTSD who have had single versus multiple exposures to traumatic experiences exhibit different clinical characteristics. For example, patients with PTSD who experienced multiple traumatic events showed greater functional impairment, longer duration and higher comorbidity with mood and anxiety disorders. ${ }^{26}$ Hence, we will employ single versus multiple incident trauma as the stratification factor in this study.

\section{Choice of comparators}

The Japanese Society for Traumatic Stress Studies published guidelines for the pharmacological treatment of patients with PTSD. ${ }^{27}$ Due to the significant lack of clinicians trained in CBT, the recommended first-line treatment involves the prescription of either paroxetine or sertraline, both of which are classified as selective serotonin reuptake inhibitors. The present study considers pharmacotherapy, clinical monitoring, psycho-education and supportive counselling, all of which are commonly used in the treatment of patients with PTSD in Japanese medical settings, as 'TAU.' 


\section{Primary hypothesis and objectives}

The primary objective of the present trial is to evaluate the hypothesis that the addition of CPT to TAU would result in outcomes that are superior to those observed for TAU alone with respect to the severity of post-traumatic stress symptoms in patients with diagnoses of PTSD, as assessed by the Clinician Administered PTSD Scale for the Diagnostic and Statistical Manual of Mental Disorders, Fifth Edition (DSM-5), (CAPS-5). The secondary objective is to test the superiority of CPT plus TAU versus TAU alone on post-traumatic stress symptoms, as assessed by patients' responses on the PTSD Checklist for DSM-5 (PCL-5) and the determination of responder status. The incidence of adverse events will also be examined to evaluate the safety of CPT for this patient population.

\section{METHODS AND ANALYSIS \\ Trial design}

The SPINET study has been designed as a 16-week, singlecentre, assessor-masked, randomised, parallel-group superiority trial that will compare the efficacy of CPT in conjunction with TAU versus TAU alone. Minimisation will be used to balance the stratification factor of traumatic event (single vs multiple/persistent) using a ratio of $1: 1$.

\section{Study setting}

The SPINET study is being conducted at the National Center of Neurology and Psychiatry (NCNP) hospital in Japan. The NCNP, located in the suburbs of Tokyo, is a national hospital specialising in the research and treatment of psychiatric, neurological, muscular and developmental disorders. The hospital mainly provides secondary or tertiary psychiatric care to socioeconomically diverse Japanese populations on both an inpatient and outpatient basis. Most new patients are referred to the NCNP hospital when usual care provided by their local institutions has proven ineffective. There is no specific department for treating PTSD at this hospital. The treatment of PTSD is usually conducted on an outpatient basis within the Department of Psychiatry and consists of pharmacotherapy, supportive therapy and/or active monitoring.

\section{Eligibility criteria}

Individuals fulfilling the following inclusion criteria will be registered to participate in the trial: (1) diagnosis of PTSD according to criteria outlined in the DSM-5, as assessed by CAPS-5; (2) aged between 18 and 70 years (inclusive) at baseline and (3) provision of written informed consent prior to participation in the study. Exclusion criteria are as follows: (1) severe substance use disorders at baseline, as assessed by the Mini-International Neuropsychiatric Interview (MINI); (2) current manic episode or psychotic disorders at baseline, as assessed by the MINI; (3) serious suicidal ideation at baseline, as assessed by the MINI; (4) severe or unstable physical disorders or major cognitive deficits at baseline; (5) involvement in manualised psychotherapy at baseline and (6) other relevant reasons for exclusion, as determined by the investigators (eg, moving away before the start of the treatment, incarceration).

\section{Interventions}

\section{Cognitive processing therapy}

The intervention group in the present study will receive CPT, a structured psychotherapy for the treatment of PTSD, following the comprehensive manual. ${ }^{13}$ Among the various formats of CPT available (eg, group, individual, with or without written trauma account), the SPINET trial will implement CPT without the use of written trauma accounts (ie, the latest version of CPT) over an intervention period of 16 weeks. Although CPT usually consists of 12 sessions, a maximum of 16 sessions will be allowed to approximate the timelines commonly used in Japanese medical settings. The number of sessions will be determined through discussions among the therapist, patient and/or supervisors in consideration of the progress of treatment, examining the progress of weekly PCL-5 scores. We consider completer status as the completion of all 12 sessions of CPT, as outlined in the comprehensive manual. ${ }^{13}$ Treatment will focus on identifying and modifying specific maladaptive cognitive beliefs and reactions that have developed owing to experiencing the traumatic event. The CPT case formulation will be based on the social-cognitive model of PTSD, which characterises PTSD as a discontinuation of the natural recovery process associated with traumatic events. In the CPT framework, such discontinuation, or the process of being 'stuck', is caused by specific, maladaptive cognitive beliefs that inhibit the patient's ability to process cognitive and emotional information regarding the traumatic event and its consequences. For example, one patient might have excessive guilt or misattribution regarding the traumatic event (eg, "I should have predicted the traumatic event and prevented it from occurring", "The event was my fault. I was wrong"). Alternatively, the patient might have overgeneralised thoughts (eg, "I have to be cautious at all times anywhere I go because the world is dangerous"). These maladaptive cognitive beliefs may contribute to the maintenance of PTSD symptoms (eg, negative mood induced by the excessive guilt or hyperarousal induced by the overgeneralisation of danger). In CPT, the patient and therapist collaborate to identify and challenge such thoughts and beliefs.

In the first session, patients are educated on the specifics of this model and asked to write an impact statement, that is, their explanation for why they think the event occurred and the event's consequences, between the first and second sessions. The patient and therapist use this impact statement to identify the issues unique to the patient and to explore the natural emotions associated with the traumatic event. Beginning in the third session, the patient uses worksheets to learn how to monitor his or her own psychological responses and to identify the activating events, beliefs and consequences 
(emotions) associated with such responses (A-B-C worksheet).

In subsequent sessions, the patient focuses on acquiring the skill of cognitive reappraisal (cognitive restructuring) through Socratic questioning by the therapist and practice using the Challenging Questions Worksheet, Patterns of Problematic Thinking Worksheet and Challenging Beliefs Worksheet. While the first half of the treatment is devoted mainly to challenging the misattributions of the causes of traumatic events (ie, self-blame, undoing), the last half of CPT is devoted to challenging the beliefs associated with the following themes: safety, trust, power/ control, esteem and intimacy.

Psychotherapists involved in the SPINET study must be licensed clinical psychologists or physicians who have undergone at least 14 hours of CPT training. In addition, these therapists must have observed the CPT sessions of at least two cases and have experience in conducting sessions for at least one case.

\section{Treatment as usual}

Following the Japanese treatment guidelines established by the Japanese Society for Traumatic Stress Studies, ${ }^{27}$ we expect that the comparison group in the present study will receive treatments that are commonly implemented in Japanese medical settings, such as pharmacotherapy involving paroxetine/sertraline, unstructured supportive psychotherapy and/or clinical monitoring. All participants will be referred by the psychiatrists who are providing the TAU. For ethical reasons, treatment by primary care physicians will not be restricted, except for systematic psychotherapy or electroconvulsive therapy, which will be prohibited. The following specifics regarding TAU will be documented in each participant's case report during each visit: frequency and duration of treatment, use of psychotropic medication, results of the clinical evaluation and direction of future treatment.

\section{Criteria for discontinuing or modifying allocated interventions}

The intervention or follow-up observation will be discontinued when any of the following occur: (1) the participant requests to be removed from the study or withdraws consent; (2) inability to contact the participant for 1 month; (3) the entire clinical trial has been discontinued and/or (4) any other reasons that the primary investigator, therapist or supervisor have deemed worthy of discontinuation (eg, hospitalisation or suicide attempt). Dates and reasons for discontinuation will be thoroughly documented in the case report form. Whenever possible, participants will be asked to participate in assessments related to the primary and secondary outcomes to determine the safety and efficacy of the interventions.

\section{Strategy and procedures for improving and monitoring adherence}

All CPT sessions will be audio and/or video recorded. Using these recordings, treatment adherence will be monitored in weekly group supervision and subsequently evaluated using the Cognitive Processing Therapy: Therapist Adherence and Competence Protocol, Individual Version-Revised. Supervisors of this study will be MH, MI and AyK, each of whom has participated in more than 48 hours of training. One-fifth of the sessions in each case will be subject to evaluations of treatment adherence and competence by other therapists in our research team. These sessions will be randomly sampled in advance of the CPT, and the therapist will not know which sessions will be assessed.

\section{Strategy for monitoring TAU}

Participants will be asked to describe the content of TAU and occurrence of any adverse events at every visit. Whenever possible, patient reports of the TAU will be verified using electronic medical records.

\section{Relevant concomitant care and interventions permitted/ prohibited during the trial}

Other forms of systematic psychotherapy or electroconvulsive therapy will be prohibited during the trial. We define 'systematic psychotherapy' as any structured psychotherapy using published treatment manuals (ie, predetermined session numbers and duration, therapeutic procedure and content). As changes in the type and/or dose of pharmacological agents may be considered within the scope of TAU, they will be permitted during the trial.

\section{Outcomes}

The CAPS- 5 will be used to assess the primary outcome measure of PTSD symptoms at 17 weeks, whereas the PCL-5 and determination of responder status will be used to assess the secondary outcomes at 17 weeks. Because there is no current standard operational definition of responder status, ${ }^{28}$ the following criteria will therefore be used to determine responder status: (1) clinical global impression improvement rating of $\leq 2$, as assessed by item 28 of the CAPS-5 and (2) loss of PTSD diagnosis, as assessed by the CAPS- 5 . An additional secondary outcome-the safety of the intervention-will also be evaluated by examining the incidence of adverse events.

\section{Other measures}

Additional measures will be included to evaluate outcomes related to depression, quality of life, functioning, dissociation and suicidal ideation. Analyses of the treatment mechanisms (ie, post-traumatic maladaptive beliefs, trauma-related guilt and emotion regulation) and treatment processes (ie, working alliance, homework compliance and treatment adherence and competence) will also be conducted.

\section{Trial timeline}

All patients who have primary physicians will be referred to this study. Participants who have provided written informed consent will be evaluated for trial eligibility. Participants confirmed eligible will be registered to 


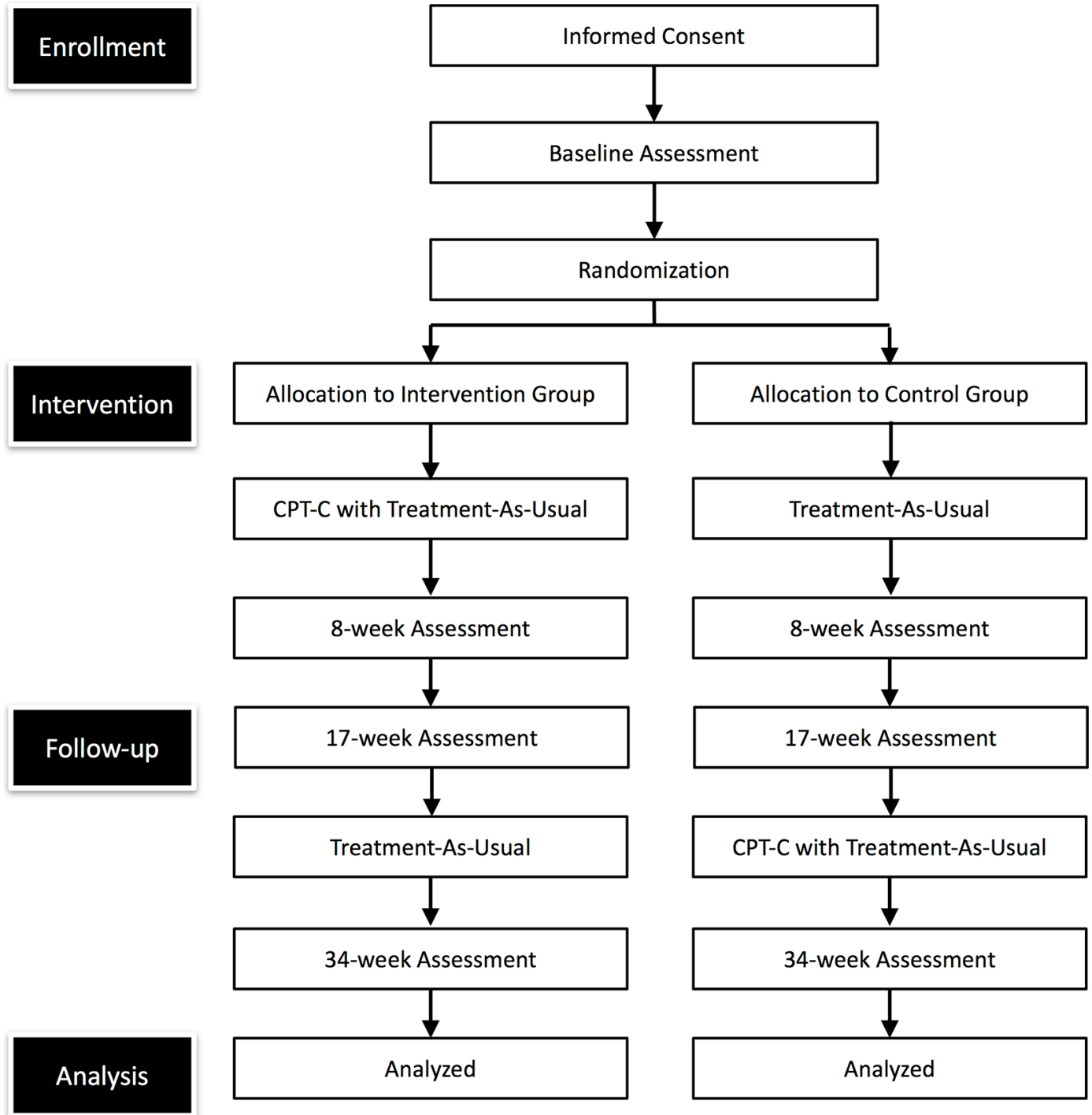

Figure 1 CONSORT flowchart of the study design. CPT, cognitive processing therapy.

the trial and randomly allocated to either the intervention or control group. The trial timeline is depicted in the CONSORT diagram (figure 1) and recommended SPIRIT template (table 1$).{ }^{29}$

\section{Sample size}

Meta-analyses have revealed that the effect size of Hedges' $\mathrm{g}$ is $1.40(95 \%$ CI 0.85 to $1.95, \mathrm{k}=4, \mathrm{n}=299)$ for CPT versus control groups (eg, TAU, wait list) with respect to post-intervention CAPS scores. ${ }^{6-8}$ A linear mixed-effects model (LMM) will be used to test the primary hypothesis (ie, combination of CPT and TAU would be superior to TAU only at the 17 week assessment). According to Diggle's formula for estimating the LMM sample size ${ }^{30}$ a minimal sample size of 3-24 participants in each group is required when assuming the following: (two sided) alpha $=0.05$; power $=0.9$; standardised mean difference $=1.40 \quad(95 \%$ CI 0.85 to 1.95 ); three assessment time-points (pre, middle, post); autoregressive covariance structure and within-subjects correlation of 0.2-0.8. Considering a drop-out rate of $22 \%,{ }^{16}$ a conservative sample size of 29 has been selected.

\section{Recruitment}

The SPINET trial will be advertised to psychiatrists and other physicians in the NCNP hospital and in collaborating clinics in the vicinity. In addition, the trial will be advertised at crime victim centres, public health centres and departments of health promotion in local governments. As there is no institute specialised for providing psychosocial interventions for patients with PTSD in the area, we expect a large number of participant referrals. Because all of the participants must be receiving TAU according to our study design, we will not accept self-referrals for this study. We estimate two registrations per month during the period between April 2016 and November 2018.

\section{Allocation}

Participants will be randomly allocated to either the intervention or the control group in a 1:1 ratio using an online, computer-generated sequence. Restricted and adapted randomisation (ie, minimisation) will be used to minimise imbalances in the types of traumatic events (single 


\begin{tabular}{|c|c|c|c|c|c|c|c|c|c|c|c|}
\hline Time points (weeks) & 0 & 1 & $2-5$ & 6 & 7 & 8 & $9-10$ & 11 & $12-16$ & 17 & 34 \\
\hline \multicolumn{12}{|l|}{ Primary and secondary outcomes } \\
\hline $\begin{array}{l}\text { Clinician-Administered PTSD Scale for } \\
\text { DSM-5 }\end{array}$ & IE & & & & & $\mathrm{IE}$ & & & & $\mathrm{IE}$ & $\mathrm{IE}$ \\
\hline $\begin{array}{l}\text { Post-traumatic Stress Disorder Checklist } \\
\text { for DSM-5 }\end{array}$ & $P$ & PtC & $\mathrm{PtC}$ & PtC & PtC & $\mathrm{P} / \mathrm{PtC}$ & PtC & PtC & PtC & $P$ & $\mathrm{P}$ \\
\hline \multicolumn{12}{|l|}{ Other outcomes (self-report) } \\
\hline Patient Health Questionnaire-9 & $P$ & PtC & PtC & PtC & PtC & $\mathrm{P} / \mathrm{PtC}$ & PtC & PtC & PtC & $P$ & $P$ \\
\hline Suicidal Ideation Attributes Scale & $P$ & PtC & $\mathrm{PtC}$ & PtC & PtC & $\mathrm{P} / \mathrm{PtC}$ & PtC & PtC & PtC & $\mathrm{P}$ & $\mathrm{P}$ \\
\hline Euro-Qol Five Dimension Five Level & $P$ & & & & & $\mathrm{P}$ & & & & $\mathrm{P}$ & $\mathrm{P}$ \\
\hline Sheehan Disability Scale & $\mathrm{P}$ & & & & & $\mathrm{P}$ & & & & $P$ & $\mathrm{P}$ \\
\hline \multicolumn{12}{|l|}{ Treatment mechanism } \\
\hline Posttraumatic Maladaptive Beliefs Scale & $\mathrm{P}$ & & & & & $\mathrm{P}$ & & & & $P$ & $P$ \\
\hline Trauma-Related Guilt Inventory & $\mathrm{P}$ & & & & & $\mathrm{P}$ & & & & $P$ & $\mathrm{P}$ \\
\hline Emotion Regulation Questionnaire & $\mathrm{P}$ & & & & & $\mathrm{P}$ & & & & $P$ & $\mathrm{P}$ \\
\hline \multicolumn{12}{|l|}{ Process measures } \\
\hline Session Rating Scale & & $\mathrm{PtC} / \mathrm{T}$ & & $\mathrm{PtC} / \mathrm{T}$ & & $\mathrm{PtC} / \mathrm{T}$ & & $\mathrm{PtC} / \mathrm{T}$ & & & \\
\hline Practice Assignment Review & & $\mathrm{T}$ & $\mathrm{T}$ & $\mathrm{T}$ & $\mathrm{T}$ & $\mathrm{T}$ & $\mathrm{T}$ & $\mathrm{T}$ & $\mathrm{T}$ & & \\
\hline Adverse Event & & $\mathrm{T}$ & $\mathrm{T}$ & $\mathrm{T}$ & $\mathrm{T}$ & $\mathrm{T} / \mathrm{IE}$ & $\mathrm{T}$ & $\mathrm{T}$ & $\mathrm{T}$ & IE & IE \\
\hline \multicolumn{12}{|l|}{ Additional assessment } \\
\hline $\begin{array}{l}\text { Mini International Neuropsychiatric } \\
\text { Interview }\end{array}$ & IE & & & & & & & & & IE & IE \\
\hline
\end{tabular}

CPT, cognitive processing therapy; DSM-5, Diagnostic and Statistical Manual of Mental Disorders, Fifth Edition; IE, assessment by Independent Evaluator; P, patient self-report; PtC, participant during CPT treatment; PTSD, post-traumatic stress disorder; T, therapist selfreport.

event vs multiple/persistent experiences). The clinical laboratory technicians of the Clinical Research Unit at the NCNP hospital, who will remain independent from the research team involved in the SPINET study, will set up the online, central randomisation computer software for sequence generation with assistance from the trial statistician (YK). The principal investigator, therapists and members of the research team will not have access to the minimisation or probability information. Allocation will be implemented by clinical research coordinators or primary investigators in front of the eligible participants using a laptop computer. Throughout the process, no patient or investigator will be able to access information that could possibly reveal the allocation.

\section{Masking}

Independent evaluators (IEs) will be masked with regard to the allocated treatment. Independent evaluators will be excluded from all staff meetings, group sessions and mailing lists and prohibited from accessing the online allocation system. The allocation will not be revealed until the final data set has been fixed. The self-reported IE Knowledge of Outcome assessment, which has been used in a number of clinical trials, will be used to examine the success (or failure) of the masking process. ${ }^{31} 32$ The IEs will offer one of three predictions regarding the allocation of each participant (intervention, control, unknown). On completion of the trial, a table containing these predictions alongside the actual allocation data will be used to calculate the masking index. ${ }^{33}$ No emergent circumstances will be assumed to unblind the allocation to the IEs because the treatment is known to the patients, therapists and primary doctors.

\section{Data collection}

Assessment of outcomes will follow the schedule presented in table 1. The details for each assessment are included as online supplementary material, and thus, the assessments are only briefly outlined below. For the primary and secondary outcomes related to PTSD symptoms and diagnosis, the monthly version of the CAPS-5 and PCL-5 will be used to assess the severity of PTSD symptoms and to diagnose cases. ${ }^{345}$ Traumatic events will be assessed using the Life Events Checklist- 5 before the administration of CAPS- $5 .{ }^{36}$ For the other aspects of clinical outcomes, the Patient Health Questionnaire-9 (PHQ-9), Suicidal Ideation Attributes Scale (SIDAS), Euro-Qol Five Dimension Five Level (EQ-5D-5L) and Sheehan Disability Scale (SDS) will be used to assess depression, suicidality, quality of life and functional impairment, respectively. ${ }^{37-40}$ To assess the treatment mechanisms focusing on the content and process of cognitive processing, the Posttraumatic Maladaptive Beliefs Scale, Trauma-Related Guilt Inventory, and Emotion Regulation Questionnaire will be 
used. ${ }^{41-43}$ For the process measures of the intervention, we will assess therapeutic alliance, homework adherence and safety by using the Session Rating Scale, ${ }^{44}$ Practice Assignment Review ${ }^{45}$ and the question set of adverse events, respectively. To assess adverse events, therapists or IEs will question patients regarding the occurrence or worsening of any symptoms during each visit. The severity of adverse events will be assessed using criteria from the Japanese version of the Common Terminology Criteria for Adverse Events (V.4.0). ${ }^{46}$ The MINI 7.0.0., which is a widely used, short, structured diagnostic interview developed to evaluate psychiatric disorders according to DSM- 5 and ICD-10 criteria, ${ }^{47}$ will be used to ascertain the exclusion criteria and other psychiatric disorders. All assessment sessions will be audio-recorded, and different IEs will evaluate one-fifth of the randomly sampled audio-recorded sessions to determine the inter-rater reliability of the CAPS-5.

\section{Plan to promote participant retention and complete follow- up}

To promote transparency, the allocation process will be conducted by the trial coordinator in front of the participant and their designated CPT therapist using a laptop computer. Participants disappointed by their allocation to the control group will receive, if necessary, minimal emotional support and assistance in engaging in their TAU from the CPT therapist who will also emphasise that the participant can start CPT 18 weeks later. The participant can then schedule their CPT appointments, which will start at week 18 . The allocation session will take about 50 min. After each assessment point, the results will be summarised and sent to participants along with motivational messages designed to promote adherence to the trial. Participants who drop out or meet any criteria for discontinuation will still be asked to participate in the week 17 assessment. This request will be emphasised to the participant at the time of informed consent. No monetary or physical incentives will be provided to patients at any point during the assessment or intervention.

\section{Data management}

All assessment and case report data will be entered into a Microsoft Access (Microsoft) database. To prevent keystroke errors, the database will be configured such that only those numbers within the range of possible scores will be accepted (eg, only entries of $1-5$ will be allowed when the range of possible scores for the item is 1-5). New data will be immediately saved to the hard drive and encrypted by the trial staff. Data entry will be conducted using a standalone computer without any connection to the Internet. Entered data will be confirmed by a second person. Only individuals who have received permission from the primary investigator will be allowed access to the database. Original paper records for each participant will be stored according to binder type (ie, paper materials related to the assessments will be stored in an assessment binder and intervention materials will be stored in a separate intervention binder). The paper binders will be stored in numerical order in a locked cabinet at the National Center for Cognitive-Behavior Therapy and Research within the NCNP. The paper files will be stored for a period of 5 years after trial completion.

\section{Statistical methods}

The statistician for this trial (YT) will analyse the data. Primary outcomes will be analysed using an LMM and autoregressive repeated covariance type. Patient scores on the CAPS- 5 will be considered dependent variables. Group allocation (intervention $=0$, control $=1$ ), assessment time point (baseline $=0$, week $8=1$, week $17=2$ ), and the interaction between group allocation and assessment time point will be regarded as fixed-effect factors, whereas participant will be regarded a random-effect factor. Statistical significance will be set at $\mathrm{p}<0.05$ for two-tailed t-tests. Analyses of secondary outcomes (eg, PCL-5) and other exploratory outcomes (PHQ-9, EQ-5D-5L, SIDAS, SDS) will be conducted in the same fashion. The proportion of individuals considered responsive to treatment will be examined using a risk ratio with a $95 \%$ CI. Safety will be evaluated using a cross-table for the occurrence of adverse events. Subgroup analyses have not been planned at this time. Up-to-date versions of $\mathrm{R}$ will be used to analyse the data. Following the intent-to-treat principle, data from all allocated participants will be used to examine the safety and efficacy of the interventions. However, secondary analysis of all data not meeting the discontinuation criteria will be conducted using a per-protocol approach. Although we consider that the LMM is capable of resolving the problem of missing values, a multiple imputation method analysis will be conducted as a sensitivity analysis. Missing values related to secondary or exploratory outcomes will be handled similarly.

\section{Data monitoring}

The primary investigators of the SPINET study, in collaboration with the study coordinators and data manager, will conduct on-site and central monitoring and will report the results to the Data and Safety Monitoring Board (DSMB). The DSMB consists of two psychiatrists who will remain independent of all activities associated with this clinical trial. On-site monitoring (ie, checking the congruence of the entered data with respect to the original case report forms/assessments) will be conducted by the data manager following a checklist developed in advance of the study. The first three registered cases along with three randomly presampled cases selected from among cases 4 to 29 will undergo on-site monitoring. Cases to undergo on-site monitoring will not be disclosed to personnel who complete the data entry and/or fill in the case report forms. If significant errors/issues are discovered during on-site monitoring, additional on-site monitoring will be conducted. All registered cases will undergo central monitoring by the trial statistician every 6 months (at the end of March and October). The primary investigators and members of the DSMB have the right to designate DSMB 
meeting locations. Members of the DSMB can request that the data manager share specific study information.

Plans for an interim analysis have not been formulated at this time. The SPINET trial will be stopped when the institutional review board (IRB) and/or DSMB recommends doing so. The primary investigators and members of the DSMB will evaluate the continuation of the trial if any of the following occur: (1) the primary investigator obtains important information regarding the quality, safety and efficacy of the intervention or (2) the IRB requests a change to the study protocol that is difficult to implement.

\section{Assessment of harm and invasiveness}

Invasiveness is defined as the occurrence of physical or mental harm or the experience of significant burden by participants due to any purposeful research act, including questioning regarding psychological trauma. ${ }^{48} 49$ Such questions will be included in the assessments (ie, CAPS-5, PCL-5) and interventions used in the SPINET study. Because these questions are routinely implemented in standard clinical practice for the treatment of PTSD and are not considered to result in permanent alterations to a patient's psychological state, we believe that the treatments used in the present study are minimally invasive. Information regarding the occurrence of adverse events will be solicited from patients according to a previously described protocol, with particular focus on suicidal ideation, as assessed using the SIDAS. Should an adverse event occur, the investigator (ie, or therapist) will assess and document the content, duration, severity, outcome and relatedness of the event to trial participation. Severe adverse events will be defined according to established ethical guidelines in Japan, as follows: death, threat to life requiring inpatient treatment, permanent or significant disorder/dysfunction or development of congenital anomaly. Details regarding severe adverse events will be immediately reported to the IRB of the NCNP. Other information related to adverse events will be reported to the DSMB every 6 months and to the IRB once per year.

\section{Auditing}

Auditing of data has not been planned at this time.

\section{ETHICS AND DISSEMINATION}

\section{Ethical approval, protocol amendments, consent}

The SPINET study has been approved by the IRB of the NCNP (approval number: A2015-111; 16 March 2016). Approval from the NCNP will be required to change the protocol, forms and documents used to obtain informed consent. Details of the SPINET study will be explained to patients prior to their participation in the study by the primary investigator or research coordinator using an informational leaflet. Written informed consent will be obtained from patients who agree to participate in the study. Informed written assent and consent will be obtained from both patients and their guardians/family members in cases where participants are under the age of 20.

\section{Confidentiality}

All data obtained will be securely stored at the NCNP. The collected paper documents will be filed separately according to participant. These files will be stored in a locked cabinet located in a doubly locked room. All identifying information associated with specific participants will be excluded from paper files. All identifying information will be recorded with a participant number to secure patient privacy, and no personal identity-related information will be entered into the electronic database. The key connecting the participant numbers and names (as well as contact and tracking information) will be kept separate from the data files. The electronic database and all other electronic data (ie, audio or video recordings) will be saved to an encrypted hard drive with a password lock. The hard drive will be stored in the locked cabinet along with the paper files. Data entry and statistical analyses will be conducted using a standalone computer without any connection to the internet or hospital intranet. All other papers with identifying information (ie, signed informed consent forms) will be stored in a separate locked cabinet.

\section{Access to data}

Prior to publication of the primary outcome paper, only the data manager will have access to the full data set. The data manager will verify any problems (eg, missing data, illogical data) in the data and finalise the data set for statistical analysis. Following publication, only the primary investigators and those individuals who receive approval from the primary investigators will have access to the cleaned data set.

\section{Ancillary and post-trial care}

During and following participation in the SPINET study, participants will be allowed to contact the trial coordinators. If any adverse events requiring additional care occur, the principal investigator will arrange appropriate medical or psychological care. Patients will not be compensated for medical costs related to harm caused by adverse events. If harm occurs, we will provide necessary treatment by using the participant's health insurance, because the occurrence of harm beyond that typically associated with the care provided in the trial is not expected.

\section{Dissemination policy}

Regardless of the results, the primary outcome will be published in a peer-reviewed journal. In addition, a summary of the results will be provided on our website to alert the participating physicians, medical staff and participants of the findings and scientific significance of the study. Exploratory analyses of the data will be conducted to examine the treatment parameters (ie, relationships between the intervention outcome and trauma-related cognition, treatment and demographic variables) and identify the sequence of symptom recovery. 


\section{DISCUSSION}

There is a substantial need for care among individuals with PTSD and related difficulties in Japan. This clinical trial is expected to provide evidence of the efficacy and safety regarding one of the most disseminated PTSD treatments, CPT, among Japanese patients with PTSD. Although we designed this trial to achieve higher scientific validity, we had to abandon the application of more rigorous conditions for conducting the trials owing to feasibility issues in managing the trial in the Japanese medical setting. Three major limitations of the study exist regarding the control conditions and participant characteristics. The first limitation is that we could not employ active control conditions, such as PE or present-centred therapy. Hence, we will not be able to conclude the superiority of CPT to the common therapeutic factors or other established treatments (ie, PE), although that is not our primary focus. Second, we will not be able to limit changes to pharmacotherapy during the course of this study because participant recruitment would be hindered by doing so in Japan. Third, the participants cannot selfrefer to the study, which limits the generalisability of the results. Despite these limitations, this study is expected to provide useful information regarding the applicability of CPT in the Japanese settings and population.

\section{Current study status}

The SPINET study began recruiting participants on 1 April 2016. Three participants have been enrolled at the time of submission of this protocol.

\section{Author affiliations \\ ${ }^{1}$ Department of Clinical Dissemination and Training, National Center for Cognitive Behavior Therapy and Research, National Center of Neurology and Psychiatry, Tokyo, Japan \\ ${ }^{2}$ National Center for Cognitive Behavior Therapy and Research, National Center of Neurology and Psychiatry, Tokyo, Japan \\ ${ }^{3}$ Psychiatry and Behavioral Sciences, Duke University, Durham, North Carolina, USA ${ }^{4}$ Department of Functional Brain Research, National Institute of Neuroscience, National Center of Neurology and Psychiatry, Tokyo, Japan \\ ${ }^{5}$ Department of Psychology, Surugadai University, Saitama, Japan \\ ${ }^{6}$ Department of Health Risk Communication, Fukushima Medical University School of Medicine, Fukushima, Japan \\ ${ }^{7}$ National Center Hospital, National Center of Neurology and Psychiatry, Tokyo, Japan \\ ${ }^{8}$ Department of Health Promotion and Human Behavior, Kyoto University Graduate School of Medicine/School of Public Health, Kyoto, Japan}

Acknowledgements The authors express their appreciation to Fumi Imamura, PhD, Akiko Kikuchi, PhD, Satomi Nakajima, MD, PhD and Nobuaki Morita, MD, PhD for their suggestions that improved CPT implementation; Yuki Oe, MA, Tomomi Narisawa MA, Rieko Konno, MA, Tomoko Masaki, MA, and Miyuki Makino, MA for their suggestions to improve the assessment and assistance in its implementation; Yutaka Ono, MD, PhD and Nobuaki Nomura, MD, PhD for their assistance in managing the Data and Safety Monitoring Board; En Kimura, MD and the staff of the cluster ward at the NCNP Hospital for organising therapy rooms; Kyoko Akutsu for constructing the database; Tamae Sato for data entry and assistance in coordinating the trial, Ichiko liduka, MA for constructing the monitoring system and Kenji Hatano, MD and Gensei Shimizu for designing the allocation system.

Contributors All authors contributed to the conception and implementation of this clinical trial. Ml and $\mathrm{MH}$ are the coprimary investigators of this trial. MI designed the trial; drafted the research protocol; organised the research team; developed the procedural manual for monitors, therapists and independent evaluators; conducted every aspect of trial management. MH directed and supervised these processes. TAF and PAR contributed the inevitable aspects of designing the trial. MI, MH, PAR and AyK trained the therapists and developed the framework for training/supervising the process. AyK and NH developed the referral system and coordinated the medical management system for this study. Ml and AkK developed the coordinator management system. MI and MM developed the training system for independent investigators and managed the training system for independent assessment. YT, TAF and MI designed the statistical analysis plan.

Funding This study is supported by a Grant-in-Aid for Scientific Research (A) (15H01979) awarded to MH from the Japan Society for the Promotion of Science and a Research and Development Grant for Comprehensive Research for Persons with Disabilities from the Japan Agency for Medical Research and Development awarded to MI (16769055). These funders had no role in designing or executing the study.

Competing interests The primary investigators ( $\mathrm{Ml}$ and $\mathrm{MH}$ ) received royalties from Sogen-sha publishers. TAF reports personal fees from Eli Lilly, Janssen,Meiji, Mitsubishi-Tanabe, MSD, Pfizer, Takeda Science Foundation, Igakushoin Publisher and Nihon Bunka Kagaku Publisher, as well as grants and personal fees from Mitsubishi-Tanabe and Mochida, outside the submitted work.

Ethics approval Ethics committee at National Center of Neurology and Psychiatry.

Provenance and peer review Not commissioned; externally peer reviewed.

Open Access This is an Open Access article distributed in accordance with the Creative Commons Attribution Non Commercial (CC BY-NC 4.0) license, which permits others to distribute, remix, adapt, build upon this work non-commercially, and license their derivative works on different terms, provided the original work is properly cited and the use is non-commercial. See: http://creativecommons.org/ licenses/by-nc/4.0/

(C) Article author(s) (or their employer(s) unless otherwise stated in the text of the article) 2017. All rights reserved. No commercial use is permitted unless otherwise expressly granted.

\section{REFERENCES}

1. National Police Agency. The situation of crime in Japan, 2014. www. npa.go.jp/english/kokusai/2015POJcontents.html. (accessed 22 Jun 2016).

2. American Psychiatric Association. Diagnostic and statistical manual of mental disorders. $5^{\text {th }}$ Edition. Washington, DC: American Psychiatric Association, 2013. DSM-5.

3. Del Gaizo AL, Elhai JD, Weaver TL, et al. Poor physical health and substance use behaviors in a national trauma-exposed sample. Psychiatry Res 2011;188:390-5.

4. Gupta MA. Review of somatic symptoms in post-traumatic stress disorder. Int Rev Psychiatry 2013;25:86-99.

5. Kawakami N, Tsuchiya M, Umeda M, et al. Trauma and posttraumatic stress disorder in Japan: results from the World Mental Health Japan Survey. J Psychiatr Res 2014;53:157-65.

6. Jonas DE, Cusack K, Forneris CA, et al. ; Psychological and Pharmacological Treatments for Adults With Posttraumatic Stress Disorder (PTSD). . Comparative effectiveness review No. 92. (Prepared by the RTI International-University of North Carolina Evidence-based Practice Center under Contract No. 290-200710056-I, . Rockville, MD, : AHRQ Publication No. 13-EHC011EFAgency for Healthcare Research and Quality, April 2013.

7. Bisson JI, Roberts NP, Andrew M, et al. Psychological therapies for chronic post-traumatic stress disorder (PTSD) in adults. Cochrane Database Syst Rev 2013;13:Cd003388.

8. Cusack K, Jonas DE, Forneris CA, et al. Psychological treatments for adults with posttraumatic stress disorder: A systematic review and meta-analysis. Clin Psychol Rev 2016;43:128-41.

9. Watts BV, Schnurr PP, Mayo L, et al. Meta-analysis of the efficacy of treatments for posttraumatic stress disorder. J Clin Psychiatry 2013;74:e541-e550.

10. Haagen JF, Smid GE, Knipscheer JW, et al. The efficacy of recommended treatments for veterans with PTSD: A metaregression analysis. Clin Psychol Rev 2015;40:184-94.

11. Meyers LL, Strom TQ, Leskela J, et al. Service utilization following participation in cognitive processing therapy or prolonged exposure therapy for post-traumatic stress disorder. Mil Med 2013;178:95-9.

12. Iverson KM, Gradus JL, Resick PA, et al. Cognitive-behavioral therapy for PTSD and depression symptoms reduces risk for future intimate partner violence among interpersonal trauma survivors. J Consult Clin Psychol 2011;79:193-202. 
13. Resick PA, Monson CM, Chard KM, et al. Cognitive processing therapy for PTSD: a comprehensive manual. New York, NY: Guilford Press, 2016.

14. Resick PA, Wachen JS, Mintz J, et al. A randomized clinical trial of group cognitive processing therapy compared with group presentcentered therapy for PTSD among active duty military personnel. $J$ Consult Clin Psychol 2015;83:1058-68.

15. Bass JK, Annan J, Mclvor Murray S, et al. Controlled trial of psychotherapy for Congolese survivors of sexual violence. $N$ Engl $J$ Med 2013;368:2182-91.

16. Resick PA, Galovski TE, O'Brien Uhlmansiek M, et al. A randomized clinical trial to dismantle components of cognitive processing therapy for posttraumatic stress disorder in female victims of interpersonal violence. J Consult Clin Psychol 2008;76:243-58.

17. Morland LA, Mackintosh MA, Greene CJ, et al. Cognitive processing therapy for posttraumatic stress disorder delivered to rural veterans via telemental health: a randomized noninferiority clinical trial. J Clin Psychiatry 2014;75:470-6.

18. Weiss WM, Murray LK, Zangana GA, et al. Community-based mental health treatments for survivors of torture and militant attacks in Southern Iraq: a randomized control trial. BMC Psychiatry 2015;15:249.

19. Bolton $\mathrm{P}$, Bass JK, Zangana GA, et al. A randomized controlled trial of mental health interventions for survivors of systematic violence in Kurdistan, Northern Iraq. BMC Psychiatry 2014;14:360.

20. van Minnen A, Hendriks L, Olff M, et al. When do trauma experts choose exposure therapy for PTSD patients? A controlled study of therapist and patient factors. Behav Res Ther 2010;48:312-20.

21. Schumm JA, Walter KH, Bartone AS, et al. Veteran satisfaction and treatment preferences in response to a posttraumatic stress disorder specialty clinic orientation group. Behav Res Ther 2015;69:75-82.

22. Larsen SE, Wiltsey Stirman S, Smith BN, et al. Symptom exacerbations in trauma-focused treatments: associations with treatment outcome and non-completion. Behav Res Ther 2016;77:68-77.

23. Asukai N, Saito A, Tsuruta N, et al. Efficacy of exposure therapy for Japanese patients with posttraumatic stress disorder due to mixed traumatic events: A randomized controlled study. J Trauma Stress 2010;23:744-50.

24. Ono Y, Furukawa TA, Shimizu E, et al. Current status of research on cognitive therapy/cognitive behavior therapy in Japan. Psychiatry Clin Neurosci 2011;65:121-9.

25. Cook JM, Dinnen S, Coyne JC, et al. Evaluation of an implementation model: a national investigation of VA residential programs. Adm Policy Ment Health 2015;42:147-56.

26. Karam EG, Friedman MJ, Hill ED, et al. Cumulative traumas and risk thresholds: 12-month PTSD in the World Mental Health (WMH) surveys. Depress Anxiety 2014;31:130-42.

27. Japanese Society for Traumatic Stress Studies. Pharmacotherapy of PTSD Guideline for Primary Care, 1st edition. 2013 http://www.jstss. org/topics/573.php (accessed 22 Jun 2016).

28. Loerinc AG, Meuret AE, Twohig MP, et al. Response rates for CBT for anxiety disorders: Need for standardized criteria. Clin Psychol Rev 2015;42:72-82.

29. Chan AW, Tetzlaff JM, Altman DG, et al. SPIRIT 2013 statement: defining standard protocol items for clinical trials. Ann Intern Med 2013;158:200-7.

30. Diggle PJ, Heagerty PJ, Liang K, et al; Analysis of Longitudinal Data. $2^{\text {nd }}$ edition: Oxford: Oxford University Press , 2002.
31. Barlow DH, Gorman JM, Shear MK, et al. Cognitive-behavioral therapy, imipramine, or their combination for panic disorder: a randomized controlled trial. JAMA 2000;283:2529-36.

32. Roll D, Ray SE, Marcus SM, et al. Independent evaluator knowledge of treatment in a multicenter comparative treatment study of panic disorder. Neuropsychopharmacology 2004;29:612-8.

33. Bang $\mathrm{H}, \mathrm{Ni}$, Davis $\mathrm{CE}$. Assessment of blinding in clinical trials. Control Clin Trials 2004;25:143-56.

34. Weathers FW, Blake DD, Schnurr PP, et al. The ClinicianAdministered PTSD Scale for DSM-5 (CAPS-5). 2013 http://www. ptsd.va.gov/professional/assessment/adult-int/caps.asp (accessed 22 Jun 2016).

35. Weathers FW, Litz BT, Keane TM, et al. The PTSD Checklist for DSM5 (PCL-5). 2013 http://www.ptsd.va.gov/professional/assessment/ adult-sr/ptsd-checklist.asp (accessed 22 Jun 2016).

36. Weathers FW, Blake DD, Schnurr PP, et al. The Life events Checklist for DSM-5 (LEC-5). 2013 http://www.ptsd.va.gov/professional/ assessment/te-measures/life events checklist.asp (accessed 22 Jun 2016)

37. Kroenke K, Spitzer RL. The PHQ-9: a New Depression Diagnostic and Severity measure. Psychiatr Ann 2002;32:509-15.

38. van Spijker BA, Batterham PJ, Calear AL, et al. The suicidal ideation attributes scale (SIDAS): Community-based validation study of a new scale for the measurement of suicidal ideation. Suicide Life Threat Behav 2014;44:408-19.

39. Ikeda $\mathrm{S}$, Shiroiwa $\mathrm{T}$, Igarashi $\mathrm{A}$, et al. Developing a japanese version of the EQ-5D-5L value set. J Nat Inst Pub Health 2015;64:47-55.

40. Sheehan DV, Harnett-Sheehan K, Raj BA. The measurement of disability. Int Clin Psychopharmacol 1996;11(Suppl 3):89-95.

41. Vogt DS, Shipherd JC, Resick PA. Posttraumatic maladaptive beliefs scale: evolution of the personal beliefs and reactions scale. Assessment 2012;19:308-17.

42. Kubany ES, Haynes SN, Abueg FR, et al. Development and validation of the Trauma-Related guilt inventory (TRGI). Psychol Assess 1996;8:428-44.

43. Gross JJ, John OP. Individual differences in two emotion regulation processes: implications for affect, relationships, and well-being. $J$ Pers Soc Psychol 2003;85:348-62.

44. Duncan BL, Miller S, Sparks JA, et al. The session Rating Scale: preliminary psychometric properties of a "working" alliance measure. $J$ Brief Ther 2003;3:3-12.

45. Resick PA, Monson CM, Chard KM, et al. Cognitive processing therapy: veteran/Military Version: therapist and Patient Materials Manual. Washington, DC: Department of Veterans Affairs, 2014.

46. US Department of Health and Human Services. Common Terminology Criteria for adverse events (CTCAE) Version 4.0. Washington, DC: National Institutes of Health-National Cancer Institute, 2009.

47. Sheehan DV, Lecrubier Y, Sheehan KH, et al. The Mini-International Neuropsychiatric Interview (M.I.N.I.): the development and validation of a structured diagnostic psychiatric interview for DSM-IV and ICD-10. J Clin Psychiatry 1998;59 Suppl 20((Suppl 20)):22-33. quiz 34-57.

48. Ministry of Education C, Sports, Science and Technology, Ministry of Health Law. ethical guidelines for Medical and Health Research Involving Human Subjects. Japan 2014 http://www.mhlw.go.jp/ file/06-Seisakujouhou-10600000-Daijinkanboukouseikagakuka/ 0000080278.pdf (accessed 22 Jun 2016).

49. Japan Clinical Oncology Group. Common Terminology Criteria for adverse events (CTCAE) Version 4.0. Japan 2009 http://www.jcog.jp/ doctor/tool/ctcaev4.html (accessed 22 Jun 2016). 